\title{
Research on Green Product Design and Sustainable Development
}

\author{
Wei $\mathrm{He}^{1, \text { a }}$ Wanxiang $\mathrm{Li}^{2, \mathrm{~b}}$ \\ ${ }^{1}$ School of Art and Design, Lanzhou Jiaotong University, Lanzhou, 730070 \\ ${ }^{2}$ School of Mechanical and Electrical Engineering, Lanzhou Jiaotong University, Lanzhou 730070
}

Keywords: Research, Green Product Design, Sustainable Development

\begin{abstract}
This paper discusses the relationship between product design and sustainable development, that green product design is one of the effective means to achieve sustainable development, in order to comply with China to create a "harmonious society, circular economy" social development model, green product design should become China an important part of the sustainable development strategy, so that we can in the 21st century to create a suitable for human survival, with high cultural value, sustainable development of living space.
\end{abstract}

\section{Introduction}

Today's thriving industrialized civilization not only changes people's production and lifestyles, but also gradually changes people's values, because people are increasingly feel the negative effects of industrialization on the future development of mankind brought about by the shackles. In the framework of the relationship between man and nature, the product is in the position of the intermediary, the traditional product design pattern of its ultimate goal is only for the basic attributes of the product, emphasizing the design of the interests of the only subject, that is completed in the product should be achieved technology, functionality, technology and market requirements, the design goals can be achieved. For a long time, although this mode of operation has promoted the social and economic progress, but its extensive development is to destroy the ecological environment, accelerate the depletion of the heavy price of resources in exchange for, has been unable to comply with contemporary social development and environmental protection demand. Human beings in the development of the economy at the same time, we must attach importance to the natural environment to maintain a coordinated sustainable development model, and thus sustainable development of the design idea has become an inevitable choice.

In 1980, the concept of "Sustainable Development" was put forward for the first time in the World Conservation Union (SIDS), which was jointly developed by the IUCN. A clear definition of "sustainable development" is given: improving the quality of human life and not exceeding the capacity to support the development of ecosystems. This marks the era of human exploration of the environment and development issues into the substantive exploration of the times.

In the process of sustainable development, the idea of sustainable development has been continuously developed and expanded to make it gradually become an important development guiding theory that can meet the needs of the contemporary people and not to meet the needs of future generations. Sustainable development is a new way of human survival and it not only involves the use of resources and environmental protection as the main environmental life, but also as the source of the development of economic life and social life. Sustainable development is a new value that people should follow and its essence lies in making full use of modern science and technology, vigorously developing green resources, continuously improving and optimizing the ecological environment, promoting the harmonious development of man and nature, the coordination of population, resources and environment, mutual improvement.

Using the idea of sustainable development to guide product design practice is the inevitable choice of modern design development. Sustainable development design not only emphasizes that people are the main body of interest, but also recognize the status of life and the interests of nature. Contemporary design culture from the material and non-material, from the product of the object of 
production and product culture of the main interaction between the full display of the design activities not only from the product object form, structure and function to design their own works, and more concerned about the ecological the needs of the various levels of the world. Therefore, we must recognize nature, respect for nature, and abandon the traditional way of production that achieves product function and cultural value in a way that damages nature.

\section{The Concept and Its Connotation of Sustainable Development}

To achieve rapid economic growth, there must be a lot of input. This kind of short-term and extensive economic behavior characterized by high input and high consumption of natural resources can achieve temporary development (high yield and high profit) and bring about economic prosperity. (1) led to people in the immediate economic development, regardless of the cost of natural resources, resulting in excessive use or even a lot of waste; (2) relative to the economic development of natural resources, not only in the long run, but in the long run, only the value of economic resources. This is because natural resources are also part of the surface environment and the large ecosystem.

Extensive economic growth is not only a large number of consumption, waste of the Earth's non-renewable resources, and resource consumption and industrial waste caused by environmental pollution is the destruction of human ecosystems will cause ecological imbalance, a direct threat to human survival. And sustainable development is a high-tech efforts to reduce the consumption of natural resources, do everything possible to save natural resources, environmental protection and natural resources to consider the overall development, is a higher level, higher quality of healthy development as little as possible into the input, more output, in the consumption of more use, less emissions.

The broadest definition of sustainable development is "the right of mankind to enjoy a healthy and productive life in a harmonious manner with nature" and "equitably meet the needs of present and future generations in development and the environment". The sustainable development is profound and informative, but it has two basic points: one emphasizes that human beings enjoy the right to life that is healthy and productive, but this should be the unity of the way of harmony with nature should not rely on people in the hands of the technology and investment, take exhaustive resources, destruction of ecological and pollute the environment to pursue the realization of the right to development; the second is to emphasize contemporary people in the creation and pursuit of modern development and consumption and strive to make their own opportunities and equal opportunities for future generations cannot allow contemporary people blindly, one-sided, selfishly in pursuit of the development and consumption of this world, and mercilessly deprived of future generations should enjoy the same development and consumption opportunities.

Therefore, people in the transformation of the traditional development model, the implementation of sustainable development strategy, it is necessary to correct the past that rely on increased investment to increase consumption to achieve the development of the model and to sacrifice the environment to increase the output of the wrong way, so that economic development less dependent on the limited resources on earth, and more with the earth's carrying capacity to achieve organic coordination.

\section{Promote the Sustainable Development of Enterprises with Green Product Design}

The continuous development of enterprises relies on the core competitiveness and value chain management, requires understanding of the product life cycle, continue to develop new products. Green design requires the design of products must be based on environmental protection indicators to choose a reasonable raw materials, structures and processes, in the manufacture and use of the process to reduce energy consumption, does not produce toxic side effects, the product is easy to disassemble and recycling, recycling materials can be used reproduction, which makes the product can be updated alternately the use of the environment does not produce any or little burden. It requires designers to have a broader sense of knowledge and responsibility for sustainable 
development of mankind, as well as a clearer design concept and a systematic design system. Green product design is the essence of environmental awareness into the product design process, and the green characteristics of the organic integration into the whole process of life cycle. With the development and progress of society, green design is not only a significant social behavior, but also an effective means of achieving significant economic benefits, will become the mainstream of future design. In the face of environmental crisis, to promote environmental protection, to maintain the sustainable development of human thought of this rise, if you want the business in the competition is no longer just a flash in the pan, to seek the continuous development of enterprises, enterprises must seize this mainstream social development, the importance of product design.

To achieve sustainable development, enterprises are required to change the traditional mode of production and management concepts, and take the road of sustainable production, that is, for each product design, material selection, production technology, production facilities, market utilization, After-sales service and disposal should have environmental awareness should have the idea of sustainable development.

In the past to determine the market competitiveness of enterprises only need to study its production costs, production cycle, product quality is enough, and now from the concept of sustainable development, but also the product manufacturing technology and the importance of the environment and the three Point to the same degree of importance to consider, that is, in order to obtain market competitiveness, enterprises must do five: low production costs, short production cycle, high product quality, high level of technology does not affect the ecological environment, that is green product design.

\section{The Formation and Connotation of Green Design}

Green design is the 20th century, the rise of the late 80s one of the design ideas. Green design extends from the product manufacturing industry to product packaging is closely related to product packaging, product promotion and product marketing links, and further expanded to the whole society of green service awareness, green cultural awareness. Green design has two characteristics: First, the design to consider the design of the entire life cycle; Second, in order to ensure the normal function of the product at the same time, designers should give priority to the object of environmental properties, from the fundamental prevention and control of environmental pollution, energy. The green design proposition of the world today is mainly about the relationship between nature, society and people's thinking in the field of product design, production and circulation.

\section{Green Product Design Method}

Life Cycle Design Method. Modern enterprises are faced with fierce competition around the world, and the product in its life cycle should have a good environment-friendly environment (Environment) has evolved into the main aspects of its competitive performance, it is this competition led to the fundamental concept of product design change. One of the most important effects is to design the product life cycle that is from the beginning of the product concept design phase to consider all aspects of the product life cycle, including the design, development, production, supply, use, until abandoned disassemble the recycling or disposal to ensure that the green properties of the product are met. The task of life cycle design is to seek the optimal use of resources throughout the life cycle to minimize or eliminate environmental pollution. Lifecycle design is a new and challenging opportunity for enterprises, namely, life cycle design will bring more competitive products to the market to meet the needs of users and society. It can even be said that product life cycle design is the most important driving force for the future of the enterprise.

Parallel Engineering Method. Concurrent engineering is a model and system approach for modern product development. It designs products and their associated processes in an integrated, parallel way, and strives to enable product developers to consider all the elements of the product lifecycle at the beginning of the design, including quality , cost, schedule and user requirements, and ultimately to optimize the product. Parallel green design compared with the traditional design, 
to achieve the information exchange and feedback between the various links in each decision can be optimized from the perspective of product life cycle to consider the problem, thus eliminating the product design process repeated changes. But also in the design process will end of the product after the end of the demolition, separation, recovery, disposal and other aspects are taken into account, so that the design of the product from the concept of the end of life to the end of the recovery process to form a closed-loop process to meet the product life cycle full green requirements.

Modular Design Method. Modular design is in a certain range of different functions or the same function of different performance, different specifications of the product on the basis of functional analysis, division and design a series of functional modules, through the module selection and combination can constitute a different product to meet the different needs of the market. Modular design can be a good solution to product specifications, design and manufacturing cycle and the contradiction between the cost of production, but also for the rapid upgrading of products, improve product quality and ease of maintenance is conducive to the product after the demolition of waste recycling to provide the necessary conditions for competitiveness. Modular product is of great significance to green design.

Disassemble Design. Disassembly is the prerequisite for product recycling and reuse cannot be disassembled products are not only effective recovery, let alone re-use. An effective way to achieve this goal is to extend the service life of the product or to make the components or materials that make up the product can be reused, while the prerequisite for reuse is the product can effectively disassemble and recover. Product disassembly performance is good or bad on the use of the product maintenance and after the effective recovery of waste, reuse and so on are of great significance.

\section{Conclusion}

To achieve sustainable development, people are required to change the traditional concept, with new ideas to guide the design, production and consumption. Now the developed countries, industrial products to pursue the pursuit of miniaturization, multi-functional, recyclable, production technology to pursue energy saving, closed-loop, etc. are towards the sustainable development of the way. Chinese resource and energy shortage is relatively short, in the international market to enhance competitiveness, we must achieve the mode of economic growth from extensive to intensive conversion, therefore, take the road of sustainable development is the inevitable development of social and economic development, and green product design is to achieve sustainable the guarantee of development.

\section{References}

[1] Song Shouxiao, Liu Zhifeng, Liu Guangfu. Material selection of green product design [J]. Mechanical Science and Technology. 1996 (01)

[2] Lai Xiu, Meng Fenglan. Product design and market economy[J]. Agricultural Mechanization Research. 1993 (02)

[3] Li Yan. Based on the sustainable development of green product design[J] .Art and Design (Theory). 2011 (02)

[4] Sun Gang, Sheng Lianxi, Guo Ping, Zhou Daowei. Green Product Design and Sustainable Development [J] .Acta Ecologica Sinica 2001 (01)

[5] Wu Zhongren. Socialist industrial management issues to explain (twenty) to do product design work [J]. Front. 1961 (10)

[6] Huang Zhaohui, Li Liangjun. Green products green tomorrow [J]. Packaging Engineering. 2002 